\title{
Padrões de distribuição das espécies de Paspalum L. (Poaceae: Panicoideae: Paniceae) ocorrentes em Pernambuco, Brasil ${ }^{1}$
}

\author{
JEFFERSON RODRIGUES MACIEL ${ }^{2,4},{\text { REGINA CÉLIA DE OLIVEIRA }{ }^{3} \text { e MARCCUS ALVES }}^{2}$
}

(recebido: 29 de maio de 2008; aceito: 24 de junho de 2009)

\begin{abstract}
Distribution patterns of species of Paspalum L. (Poaceae: Panicoideae: Paniceae) in Pernambuco, Brazil). With an aim to contribute to our knowledge of distribution patterns of Paspalum species, this study examines the distribution of the species that occur in Pernambuco. In order to do this, extensive reviews of the literature, of specimens deposited in several herbaria, and of databases available on the Internet, in addition to collections made in the State of Pernambuco were performed. The 31 species analyzed were classified into two distribution patterns: broad, with 29 species and restricted, with two species. The species with restricted distribution are found in the Northeast region of Brazil, with one endemic to the semi-arid domain and the other until now only recorded from the Atlantic coast of Brazil. The broad distribution pattern can be subdivided into two groups, continuous and disjunct, which can be subsequently classified into three subgroups: American, Central-South American, and South American. The importance of this analysis for a better understanding of flora of Pernambuco origins is also discussed.
\end{abstract}

Key-words - endemism, Gramineae, grasses, Pernambuco

RESUMO - (Padrões de distribuição das espécies de Paspalum L. (Poaceae: Panicoideae: Paniceae) ocorrentes em Pernambuco, Brasil). A fim de contribuir para o conhecimento dos padrões de distribuição de Paspalum, este estudo trata da distribuição das espécies que ocorrem em Pernambuco. Para isto foi realizada uma intensa revisão de literatura, de coleções depositadas em diversos herbários e de bancos de dados disponíveis na internet, além de coletas no estado de Pernambuco. As 31 espécies analisadas foram classificadas em dois padrões de distribuição: amplo, com 29 espécies e restrito, com duas espécies. As espécies do padrão restrito têm sua distribuição na região Nordeste do Brasil, sendo uma endêmica ao domínio do semi-árido e outra, até o momento só registrada para a costa atlântica do Brasil. O padrão de distribuição amplo pode ser subdividido em dois grupos, contínuo e disjunto, os quais por sua vez foram classificados em três subgrupos: americano, centro-sulamericano e sulamericano. Discute-se a importância desta análise para melhor compreender as origens da flora pernambucana.

Palavras-chave - endemismo, Gramineae, gramíneas, Pernambuco

\section{Introdução}

Paspalum L. é um dos maiores gêneros da família Poaceae e seus táxons, cerca de 330, são nativos da América tropical e subtropical, e alguns ocorrem no Velho Mundo. O gênero é caracterizado pelas espiguetas plano-convexas, adaxiais, distribuídas unilateralmente na ráquis e a ausência de gluma inferior na maioria das espécies (Clayton \& Renvoize 1986). Atualmente são reconhecidos quatro subgêneros: Paspalum subg. Anachyris Chase, $P$. subg. Ceresia (Pers.) Rchb., P. subg. Harpostachys (Trin.) S. Denham e P. subg. Paspalum, sendo este último o maior e dividido em cerca de 24

1. Parte da dissertação de mestrado do primeiro autor. Programa de Pós-Graduação em Biologia Vegetal-PPGBV da Universidade Federal de Pernambuco.

2. Universidade Federal de Pernambuco - UFPE, Laboratório de Morfo-Taxonomia Vegetal-MTV. Av. Moraes Rego, s/n, CDU, 50670-930 Recife, Pernambuco, Brasil.

3. Universidade Federal Rural do Semi-Árido - Ufersa, Departamento de Ciências Vegetais, Caixa Postal 137, 59700-970 Mossoró, RN.

4. Autor para correspondência: jeff.r.maciel@gmail.com grupos informais (Chase 1929, Denham 2005). Os grupos informais de $P$. subg. Paspalum foram estabelecidos com base em similaridades fenéticas.

A maioria absoluta dos táxons que compõem o gênero ocorre entre as latitudes $40^{\circ} \mathrm{N}$ e $40^{\circ} \mathrm{S}$, estando quase todas as espécies concentradas nos limites tropicais americanos, sendo o Cerrado o centro de diversidade (Chase 1929, Judziewicz 1990, Zuloaga \& Morrone 2005, Rua 2006).

Muito embora o gênero apresente uma distribuição ampla, muitas espécies possuem padrões de distribuição restrito. Nesse sentido são encontrados exemplos de espécies ou grupos de espécies endêmicos aos Andes, Cerrado, Costa Leste dos Estados Unidos, Caribe, entre outras regiões (Chase 1929, Cialdella et al. 1995, Morrone et al. 2000, Denham et al. 2002).

No Estado de Pernambuco o gênero está representado por 32 espécies, sendo que apenas uma delas não é nativa. Para Andrade-Lima (1960), Pernambuco é um estado composto principalmente por continuações ou repetições de grandes regiões fitogeográficas do Brasil, o que reflete na distribuição das espécies que compõem sua flora. 
Este estudo tem por objetivo analisar e discutir os padrões de distribuição das espécies de Paspalum que ocorrem em Pernambuco, permitindo ampliar o conhecimento sobre as rotas migratórias das espécies deste gênero e sobre as origens da flora pernambucana.

\section{Material e métodos}

Realizaram-se coletas no período de janeiro de 2006 a julho de 2007 em todas as zonas fitogeográficas do Estado de Pernambuco como definidas por Andrade-Lima (1960), a saber, zona do litoral, da mata, das caatingas e das savanas. A espécie Paspalum notatum Alain ex Flüggé foi excluída da análise por não ser nativa. As informações sobre distribuição geográfica das demais 31 espécies (tabela 1) foram coletadas no banco de dados do Catalogue of New World Grasses (CNWG 2006), nas revisões do gênero (Chase 1929 e manuscrito não publicado, Denham 2005, Morrone et al. 1996, R. C. Oliveira, dados não publicados, Zuloaga \& Morrone 2005) e nas análises das coleções depositadas nos herbários BOTU, CPATSA, CEN, HST, IBGE, ICN, IPA, JPB, MOSS, PACA, PEUFR, RB, SP, SPF, UB e UFP (Siglas conforme Holmgren et al. 2003, exceto CPATSA, Herbário da Embrapa Semi-Árido, e HST, Herbário Sérgio Tavares da Universidade Federal Rural de Pernambuco). Quando necessário foram consultadas floras para complementar os dados de distribuição.

Os mapas bases utilizados nas ilustrações foram elaborados a partir da base cartográfica do Enviromental Systems Research Institute (ESRI), e as distribuições das espécies foram plotadas nos mapas com o auxílio do Google Maps. A partir de então foram elaborados os polígonos que representam os padrões de distribuição das espécies.

\section{Resultados e discussão}

As 31 espécies analisadas neste estudo foram enquadradas em dois padrões de distribuição geográfica: ampla e restrita (tabela 1).

A distribuição ampla pode ser contínua ou disjunta. Foram ainda reconhecidas para este caso a distribuição Americana (figuras 1, 3h), a qual conta com espécies ocorrendo desde os Estados Unidos até a Argentina; a Centro-sulamericana (figuras 2, 3a-b, 4a-d), representada por espécies que ocorrem desde a região central do México até a Argentina; e a Sul-americana (figuras $3 \mathrm{c}-\mathrm{g}, 4 \mathrm{e})$, onde o limite de distribuição das espécies é o continente sul-americano.

A maioria das espécies tratadas aqui se enquadrou na distribuição ampla, apenas Paspalum divergens (figura 4f) e P. scutatum (figura 4g) apresentaram distribuição restrita, sendo ambas endêmicas do nordeste do Brasil. Resultado semelhante foi encontrado para as espécies de Phyllanthus L. (Phyllanthaceae) ocorrentes em Pernambuco (M. J. Silva, dados não publicados).

As espécies enquadradas no padrão amplo apresentaram uma predominância na distribuição contínua, o que representou 23 (79\%) das 29 espécies registradas na distribuição ampla. As seis espécies restantes mostraram padrão de distribuição disjunto (tabela 1), sendo a predominância de disjunções no padrão centro-sulamericano.

Vale destacar as disjunções apresentadas por Paspalum clavuliferum (figura 4a), P. fimbriatum (figura 4b), P. molle (figura 4c) e P. pleostachyum (figura 4d). Essas quatro espécies apresentam registros na América Central, Norte da América do Sul e Brasil. Paspalum fimbriatum, $P$. molle e P. pleostachyum no Brasil, só ocorrem na região Nordeste, já $P$. clavuliferum apresenta uma distribuição que se estende pelo Cerrado no CentroOeste e Sudeste. Disjunção desta natureza está mais relacionada à dispersão por longa distância do que por eventuais dinâmicas de massas vegetacionais (Gentry 1982). Isto é corroborado por Davidse (1986), que aponta alguns caracteres em Paspalum que o condicionaria a dispersão por longas distâncias.

Segundo Greller (2000), a província fitogeográfica da América Central possui savanas onde predominam diversos gêneros de gramíneas, entre eles Paspalum. Tais savanas são mais comuns no sudeste do México e se tornam mais raras na costa do Pacífico. Diante das características fisionômicas ressaltadas por esse autor nota-se uma relativa semelhança de fatores ecológicos entre a província fitogeográficas da América Central e as regiões da América do Sul, onde as espécies aqui estudadas são encontradas.

Dentre os padrões encontrados para a distribuição ampla, a distribuição Americana é a que apresenta o maior limite latitudinal. Neste agrupamento estão Paspalum conjugatum (figura 1a), P. denticulatum (figura 1b), P. distichum (figura 1c), P. millegrana (figura 1d), P. paniculatum (figura 1e), P. plicatulum (figura 1f), P. repens (figura 1g), P. vaginatum (figura 1h) e $P$. convexum (figura 3h), a única com distribuição disjunta. Ritter \& Waechter (2004) constataram que as espécies de Mikania Willd. com padrões de distribuição amplos, semelhantes aos das espécies de Paspalum citadas acima, foram as que apresentaram maior capacidade de adaptação.

A distribuição Centro-Sulamericana abarcou 14 espécies (49\%), sendo que 10 tiveram distribuição contínua (figuras 2a-h, 3a-b) e quatro, disjunta (figura 4a-d). Esta observação aponta para a constatação de que Paspalum é um grupo tipicamente tropical (Judziewicz 
Tabela 1. Padrões de distribuição geográfica das espécies de Paspalum que ocorrem em Pernambuco, Brasil.

Table 1. Patterns of the geographic distribution of the Paspalum species occurrents in Pernambuco, Brazil.

\begin{tabular}{|c|c|c|c|}
\hline \multirow[t]{6}{*}{ Distribuição ampla } & Contínua & Distribuição americana & $\begin{array}{l}\text { P. conjugatum } \text { P. J. Bergius } \\
\text { P. denticulatum Trin. } \\
\text { P. distichum } \mathrm{L} \text {. } \\
\text { P. millegrana } \text { Schrad. } \\
\text { P. paniculatum } \text { L. } \\
\text { P. plicatulum Michx. } \\
\text { P. repens } \text { P. J. Bergius } \\
\text { P. vaginatum Sw. }\end{array}$ \\
\hline & & Distribuição centro-sulamericana & $\begin{array}{l}\text { P. corcovadense Raddi } \\
\text { P. coryphaeum Trin. } \\
\text { P. gardnerianum Nees } \\
\text { P. melanospermum Desv. ex Poir. } \\
\text { P. multicaule Poir. } \\
\text { P. nutans Lam. } \\
\text { P. orbiculatum Poir. } \\
\text { P. parviflorum Rhode ex Flüggé } \\
\text { P. pilosum Lam. } \\
\text { P. virgatum L. }\end{array}$ \\
\hline & & Distribuição sulamericana & $\begin{array}{l}\text { P. atratum Swallen } \\
\text { P. calliferum S. Denham } \\
\text { P. maritimum Trin. } \\
\text { P. oligostachyum Salzm. ex Steud. } \\
\text { P. pumilum Nees }\end{array}$ \\
\hline & Disjunta & Distribuição americana & P. convexum Humb. \& Bonpl. ex Flüggé \\
\hline & & Distribuição centro-sulamericana & $\begin{array}{l}\text { P. clavuliferum C. Wright } \\
\text { P. fimbriatum Kunth } \\
\text { P. molle Poir. } \\
\text { P. pleostachyum Döll }\end{array}$ \\
\hline & & Distribuição sulamericana & P. arenarium Schrad. \\
\hline Distribuição restrita & & & $\begin{array}{l}\text { P. divergens Döll } \\
\text { P. scutatum Nees ex Trin. }\end{array}$ \\
\hline
\end{tabular}

1990), embora muitas de suas espécies tenham uma distribuição que ultrapassa as fronteiras dos trópicos (Chase 1929; Zuloaga \& Morrone 2005), o que já foi observado para outros gêneros de gramíneas como Eragrostis Wolf e Aristida L. (Boechat \& Longhi-Wagner 2000; Longhi-Wagner 1990).

Por fim estão as espécies com distribuição sulamericana, que são Paspalum arenarium (figura 4e), P. maritimum (figura 3e), P. oligostachyum (figura 3f), $P$. atratum (figura 3c), P. calliferum (figura 3d) e P. pumilum (figura $3 \mathrm{~g}$ ). As três primeiras possuem distribuição costeira sendo encontradas com abundância na Mata Atlântica e ecossistemas associados. Paspalum arenarium apresenta uma disjunção entre a costa brasileira e a Bolívia. Eventualmente essas espécies são encontradas em paisagens de exceção da caatinga em condições edafoclimáticas semelhantes àquelas dos ambientes costeiros. Tal comportamento é verificado para espécies de outras famílias como Phyllanthus klotzschianus Müll. Arg. (M. J. Silva, dados não publicados).

Destaca-se aqui que, os padrões encontrados para estas espécies, também foram verificados para representantes de Inga Mill. (Leguminoseae), Ouratea Aubl. (Ochnaceae), Macoubea Aubl. e Couma Aubl. (Apocynaceae) que ocorrem nas Guianas (Poncy 1991, Allorge \& Sastre 1991). Estes padrões revelam possíveis 

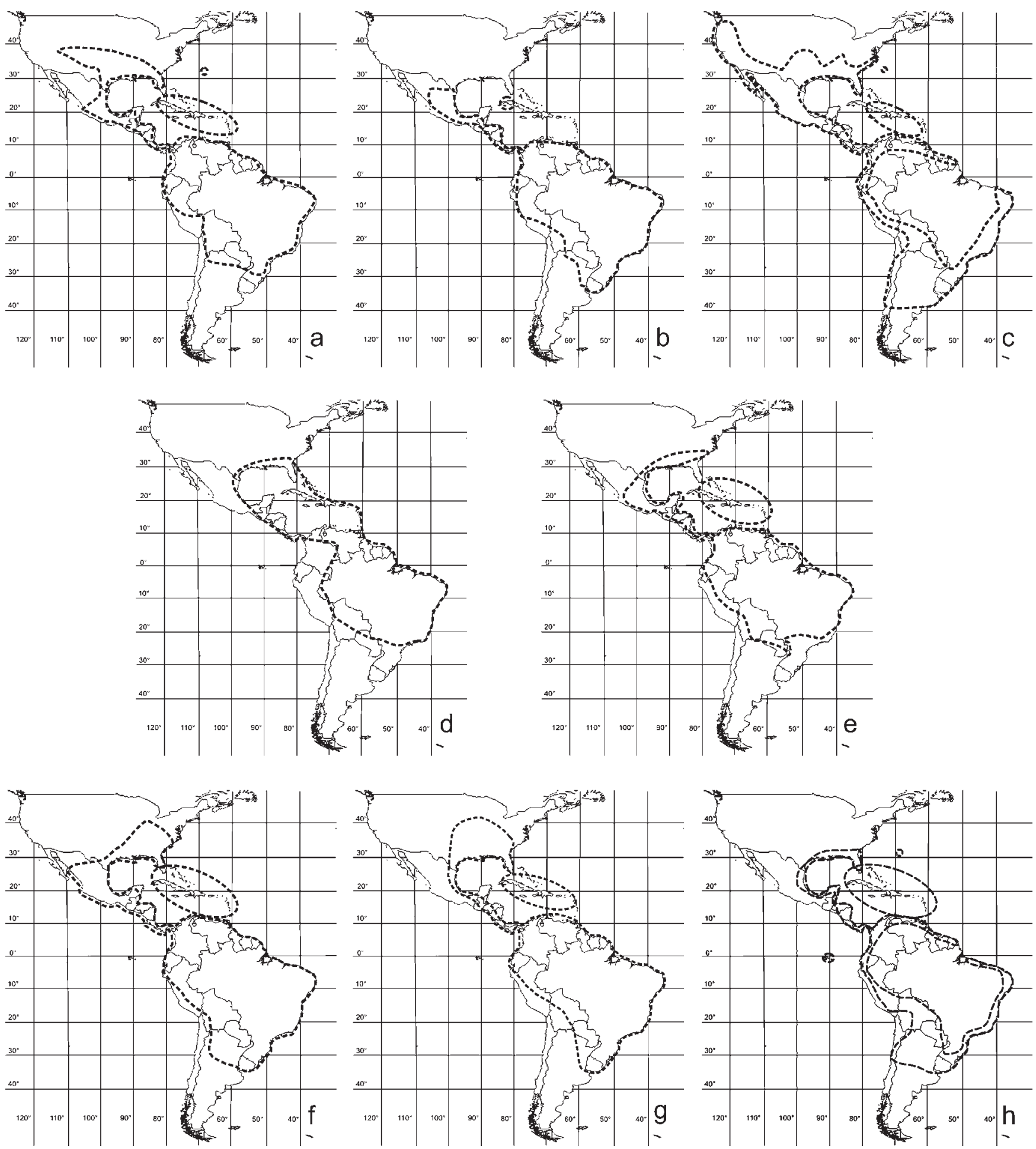

Figura 1. Padrões de distribuição das espécies de Paspalum ocorrentes em Pernambuco. a-h. Padrão amplo contínuo. Distribuição americana. a. Paspalum conjugatum. b. P. denticulatum. c. P. distichum. d. P. millegrana. e. P. paniculatum. f. P. plicatulum. g. P. repens. h. P. vaginatum.

Figure 1. Distribution patterns of species of Paspalum in Pernambuco. a-h. Broad continuous pattern. American distribution. a. Paspalum conjugatum. b. P. denticulatum. c. P. distichum. d. P. millegrana. e. P. paniculatum. f. P. plicatulum. g. P. repens. h. P. vaginatum. 

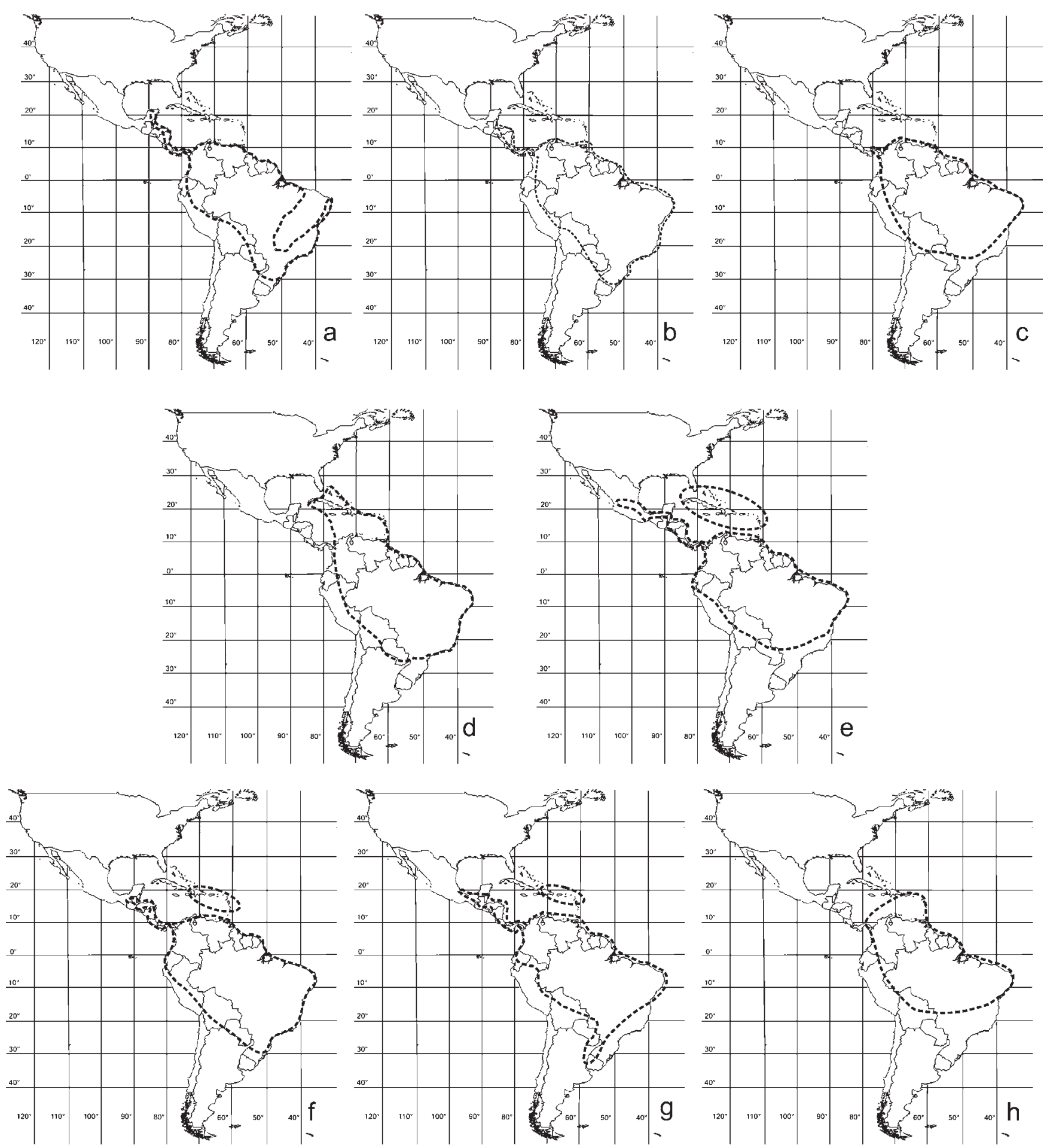

Figura 2. Padrões de distribuição das espécies de Paspalum ocorrentes em Pernambuco. a-h. Padrão amplo contínuo. Distribuição centro-sulamericana. a. Paspalum corcovadense. b. P. coryphaeum. c. P. gardnerianum. d. P. melanospermum. e. P. multicaule. f. P. nutans. g. P. orbiculatum. h. P. parviflorum.

Figure 2. Distribution patterns of species of Paspalum in Pernambuco. a-h. Broad continuous pattern. Central-southamerican distribution. a. Paspalum corcovadense. b. P. coryphaeum. c. P. gardnerianum. d. P. melanospermum. e. P. multicaule. f. P. nutans. g. P. orbiculatum. h. P. parviflorum. 

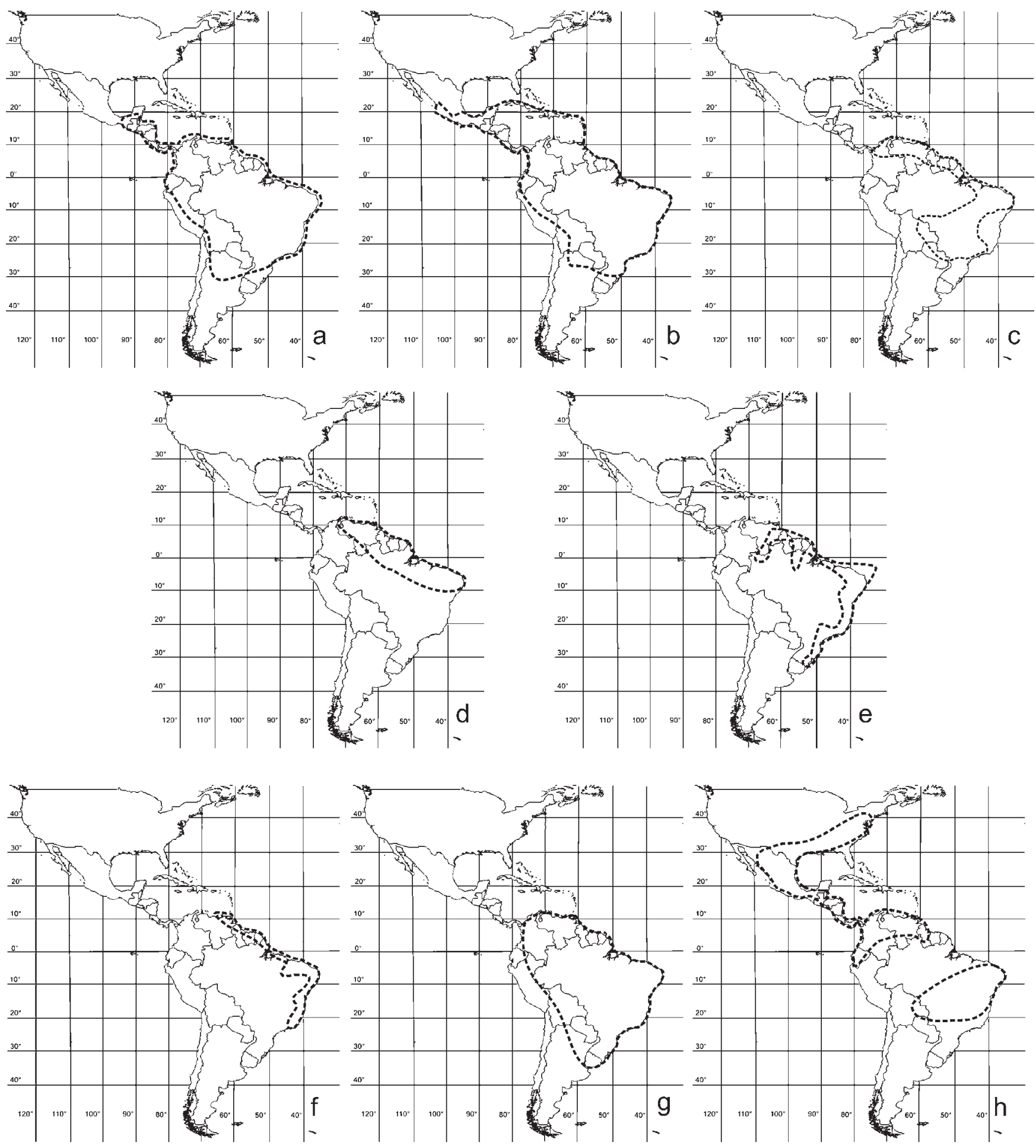

Figura 3. Padrões de distribuição das espécies de Paspalum ocorrentes em Pernambuco. a-g. Padrão amplo contínuo. a-b. Distribuição centro-sulamericana. a. Paspalum pilosum. b. P. virgatum. c-g. Distribuição sulamericana. c. Paspalum atratum. d. P. calliferum. e. P. maritimum. f. P. oligostachyum. g. P. pumilum. h. Padrão amplo disjunto. Distribuição americana. Paspalum convexum.

Figure 3. Distribution patterns of species of Paspalum in Pernambuco. a-g. Broad continuous pattern. a-b. Central-southamerican distribution. a. Paspalum pilosum. b. P. virgatum. c-g. Southamerican distribution. c. Paspalum atratum. d. P. calliferum. e. P. maritimum. f. P. oligostachyum. g. P. pumilum. h. Broad disjunct pattern. American distribution. Paspalum convexum. 

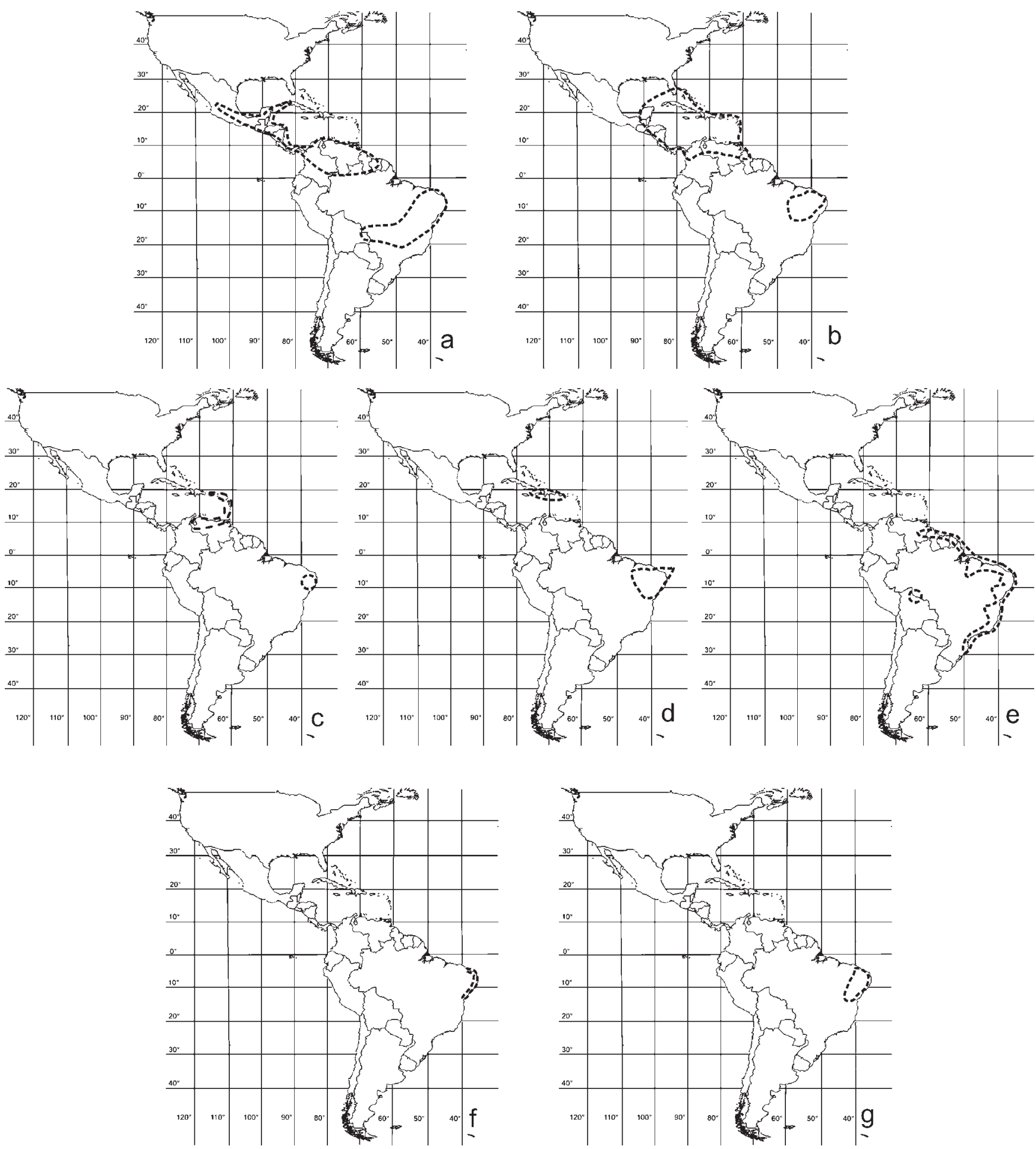

Figura 4. Padrões de distribuição das espécies de Paspalum ocorrentes em Pernambuco. a-e. Padrão amplo disjunto. a-d. Distribuição centro-sulamericana. a. Paspalum. clavuliferum. b. P. fimbriatum. c. P. molle. d. P. pleostachyum. e. Distribuição sulamericana. Paspalum arenarium. f-g. Padrão restrito. f. Paspalum divergens. g. P. scutatum.

Figure 4. Distribution patterns of species of Paspalum in Pernambuco. a-e. Broad disjunct pattern. a-d. Central-southamerican distribution. a. Paspalum. clavuliferum. b. P. fimbriatum. c. P. molle. d. P. pleostachyum. e. Southamerican distribution. Paspalum arenarium. f-g. Restrict pattern. f. Paspalum divergens. g. P. scutatum. 
conexões florísticas importantes entre as floras das Guianas e a flora da região costeira de Pernambuco. Esta observação é reforçada ao analisar o trabalho de Judziewicz (1990), que mostra 23 espécies comuns de Paspalum, entre Pernambuco e as Guianas, sendo a maioria delas das regiões litorâneas.

Diversas espécies abordadas neste estudo mostraram distribuições semelhantes às encontradas por Prado \& Gibbs (1993) para táxons lenhosos. Segundo Prado (2000), o arco pleistocênico justificaria o estabelecimento de uma unidade fitogeográfica formal cujo paradigma seria a distribuição de Anadenanthera colubrina (Vell.) Brenan (Leguminosae). Esta unidade fitogeográfica possui três núcleos onde se concentram as distribuições de várias espécies, um deles é o núcleo das Caatingas. Entre as espécies aqui estudadas, Paspalum scutatum é o único endemismo do arco pleistocênico e se restringe ao núcleo das Caatingas. Vale ressaltar que é a primeira citação desta espécie como endêmica à caatinga.

Daly \& Mitchell (2000) indicaram que grandes regiões de florestas úmidas podem ter formações anômalas com mosaicos de condições edáficas que definem a diversidade beta. São nessas formações anômalas que algumas espécies de Paspalum que ocorrem na floresta amazônica encontram condições propícias para o estabelecimento de suas populações. Isto justifica a ocorrência de algumas espécies mais típicas de ambientes abertos na região amazônica.

As fitocórias identificadas para o Norte da América do Sul por Daly \& Mitchell (2000) correspondem uma das regiões onde muitas das espécies aqui tratadas convergiram suas distribuições. Merecem destaque principalmente as fitocórias de Magdalena/NW Caribenho, dos Llanos da Venezuela e Colômbia e a porção norte da fitocória da Região Andina. Gentry (1982) ressaltou que táxons adaptados a áreas secas também são bem representados no norte venezuelano-colombiano e na região cerrado-chaco-caatinga. Alves et al. (2003) sugeriram para algumas espécies de Hypolytrum Rich. (Cyperaceae), que a fitocória Magdalena/NW Caribenho propicia condições geográficas para rotas migratórias no norte-andino.

As fitocórias de Daly \& Mitchell (2000) do norte da América do Sul correspondem em parte à província Guajira de Cabrera \& Willink (1980), região que teve sua importância para a origem da flora da caatinga destacada por Prado (2003). As evidências ressaltadas com a distribuição de algumas espécies de Paspalum reforçam e ampliam a importância do norte da América do Sul como um dos principais pontos de conexão florística com o nordeste brasileiro.
A Província das Antilhas também reuniu uma grande assembléia de espécies, tendo inclusive Paspalum pleostachyum mostrado uma ocorrência disjunta entre esta província e a região Nordeste do Brasil. O compartilhamento de elementos florísticos entre Pernambuco e a província das Antilhas pode ser explicado pelas condições climáticas relativamente semelhantes, como pode se observar nos trabalhos de Andrade-Lima (1981) e Greller (2000).

Com base nos padrões apresentados notou-se que o Istmo do Panamá se demonstrou como importante corredor migratório para as espécies de Paspalum estudadas e a província da América Central. Gentry (1982) destacou o fechamento do Istmo como um dos três eventos fundamentais para a explicação da diversidade nos neotrópicos, assim como a orogênese dos Andes. Esta região, e principalmente a porção norte, apresentou uma grande concentração das espécies com distribuição americana e centro-sulamericana aqui estudadas.

A análise dos padrões de distribuição das espécies em escala continental concorda com a proposição de Andrade-Lima (1960) de que a flora do Estado de Pernambuco compõe-se em grande parte de continuações ou repetições de grandes zonas fitogeográficas do Brasil. Por fim, é possível sugerir, com base nos dados apresentados, que os elementos que compõem a flora de Paspalum em Pernambuco e de estados adjacentes são formados sobretudo por espécies megatérmicas, com distribuição desde a América Central, porém com alguns elementos endêmicos como Paspalum divergens e P. scutatum.

Agradecimentos - Os autores agradecem aos curadores dos herbários visitados em particular a Dra. Rita de Cássia Araújo Pereira (IPA) e Marlene Barbosa (UFP) que gentilmente permitiram o acesso a estrutura dos herbários IPA e UFP para a condução da maior parte deste estudo; as Dras. Silvia Terezinha Sfoggia Miotto e Reyjane Patrícia de Oliveira assim como aos dois revisores anônimos pelos valiosos comentários. O primeiro autor agradece a concessão de Bolsa de Mestrado do Programa Taxonomia CNPq/MCT. Este estudo contou com o apoio financeiro parcial da Fundação O Boticário de Proteção à Natureza.

\section{Referências bibliográficas}

ALLORGE, L.\&SASTRE, C. 1991. Distribution geographique dans les Guyanes, d'Apocynaceae et d'Ochnaceae. Essai de mise en evidence de centres de speciation. Compterendu des Séances de la Societé de Biogeographie 67: 77-94. 
ALVES, M., THOMAS, W.W., \& WANDERLEY, M.G.L. 2003. Padrões de distribuição geográfica das espécies neotropicais de Hypolytrum Rich. (Cyperaceae). Boletim de Botânica da Universidade de São Paulo 21:265-276.

ANDRADE-LIMA, D. 1960. Estudos fitogeográficos de Pernambuco. Arquivos do IPA 5:305-341.

ANDRADE-LIMA, D. 1981. The Caatingas dominium. Revista Brasileira de Botânica 4:149-153.

BOECHAT, S.C. \& LONGHI-WAGNER, H.M. 2000. Padrões de distribuição geográfica dos táxons brasileiros de Eragrostis (Poaceae, Chloridoideae). Revista Brasileira de Botânica 23:177-194.

CABRERA, A.L. \& WILLINK, A. 1980. Biogeografia de America Latina. 2nd ed. Serie Biología. Secretaria General da Organización de los Estados Americanos, Washington.

CHASE, A. 1929. The north american species of Paspalum. Contributions from the United States National Herbarium 28:1-310.

CiAldELlA, A.M., MORRONE, O. \& ZULOAGA, F.O. 1995. Revisión de las espécies del género Paspalum (Poaceae: Panicoideae: Paniceae), grupo Bonplandiana. Darwiniana 33:67-95.

CLAYTON, W.D. \& RENVOIZE, S.A. 1986. Genera graminum: grasses of the world. Kew Bulletin Aditional Series, 13. Her Magesty's Stationary Office, London.

CNWG. CATALOGUE OF NEW WORLD GRASSES. Disponível em http://mobot.mobot.org/W3T/Search/ nwgc.html. acessado em 11/2006.

DALY, D.C. \& MITCHELL, J.D. 2000. Lowland vegetation of tropical South America. In Imperfect balance, landscapes transformation in Precolumbian Americas (D. Lentz, org.). Columbia University Press, New York., p.391-453.

DAVIDSE, G. 1986. Fruit dispersal in the Poaceae. In Grass systematics and evolution (T.R. Soderstrom, K.W. Hilu, C.S. Campbell, \& M.E. Barkworth, eds). Smithsonian Institution Press, Washington, p.143-155.

DENHAM, S.S. 2005. Revisión sistemática del subgénero Harpostachys de Paspalum (Poaceae: Panicoideae: Paniceae). Annals of the Missouri Botanical Garden 92:463-532.

DENHAM, S., ZULOAGA, F.O. \& MORRONE, O. 2002. Systematic revision and phylogeny of Paspalum subgenus Ceresia (Poaceae: Panicoideae: Paniceae). Annals of the Missouri Botanical Garden 89:337-399.

GENTRY, A. 1982. Neotropical floristic diversity: phytogeographical connections between Central and South America, pleiostocene climatic fluctuations, or an accident of the andean orogeny? Annals of the Missouri Botanical Garden 69:557-593.
GRELLER, A. 2000. Vegetation in the floristic regions of North and Central America. In Imperfect balance, landscapes transformation in Precolumbian Americas (D. Lentz, org.). Columbia University Press, New York, p.39-87.

HOLMGREN, P.K., HOLMGREN, N.H. \& BARNETT, L.C. (eds.). 2005. Index Herbariorum Part I: the herbaria of theWorld. New York, New York Botanical Garden. Disponível em: http://www.nybg.org/bsci/ih. acesso em 21/12/2006.

JUDZIEWICZ, E.J. 1990. Family 187. Poaceae (Gramineae). In Flora of the Guianas. Series A: Phanerogams. (A.R.A., Görts-van Rijn, ed.). Koltz Scientific Books, Königstein, v.8. p.1-727.

LONGHI-WAGNER, H.M. 1990. Diversidade e distribuição geográfica das espécies de Aristida L. (Gramineae) ocorrentes no Brasil. Acta Botanica Brasilica 4:105124.

MORRONE, O., DENHAM, S.S., ALISCIONI, S.S. \& ZULOAGA, F.O. 2000. Revisión de las especies de Paspalum (Panicoideae: Paniceae), subgénero Anachyris. Candollea 55:105-135.

MORRONE, O., VEGA, A.S. \& ZULOAGA, F.O. 1996. Revisión de las especies del género Paspalum L. (Poaceae: Panicoideae: Paniceae), grupo Dissecta (s. str.). Candollea 51:103-138.

PONCY, O. 1991. Le genre Inga dans les Guyanes: notes phytogeographiques. Compte-rendu des Séances de la Societé de Biogeographie 67:95-111.

PRADO, D. 2000. Seasonally dry forest of tropical South America: from forgotten ecossystem to a new phytogeography unit. Edinburgh Journal of Botany 57:437-461.

PRADO, D. 2003. As caatingas da América do Sul. In Ecologia e conservação da Caatinga. (I. Leal, M. Tabarelli, \& J.M.C. Da Silva, orgs.). Editora Universitária-UFPE, Recife, p.3-74.

PRADO, D. \& GIBBS, P.E. 1993. Patterns of species distributions in the dry seasonal forests of South America. Annals of the Missouri Botanical Garden 80:902-927.

RITTER, M. \& WAECHTER, J.L. 2004. Biogeografia do gênero Mikania Willd. (Asteraceae) no Rio Grande do Sul, Brasil. Acta Botanica Brasilica 18:643-652.

RUA, G.H. 2006. Estudos filogenéticos em Paniceae: os casos de Paspalum e Digitaria. In Os avanços da Botânica no início do século XXI: morfologia, fisiologia, taxonomia, ecologia e genética. (J.E.A. Mariath \& R.P. Santos, orgs.). Sociedade Botânica do Brasil, Porto Alegre, p.170-173.

ZULOAGA, F.O. \& MORRONE, O. 2005. Revisión de las especies de Paspalum para América del Sur Austral (Argentina, Bolívia, Sur del Brasil, Chile, Paraguay y Uruguay). Monographs in Systematic Botany from Missouri Botanical Garden 102:1-297. 